\title{
A model of HIV/AIDS population dynamics including ARV treatment and pre-exposure prophylaxis
}

\author{
Mozart U Nsuami and Peter J Witbooi*
}

"Correspondence:

pwitbooi@uwc.ac.za

Department of Mathematics and

Applied Mathematics, University of the Western Cape, Private Bag X17, Bellville, 7535, Republic of South Africa

\section{严 Springer}

\begin{abstract}
Antiretroviral treatment (ART) and oral pre-exposure prophylaxis (PrEP) have recently been used efficiently in management of HIV infection. Pre-exposure prophylaxis consists in the use of an antiretroviral medication to prevent the acquisition of HIV infection by uninfected individuals. We propose a new model for the transmission of HIV/AIDS including ART and PrEP. Our model can be used to test the effects of ART and of the uptake of PrEP in a given population, as we demonstrate through simulations. The model can also be used to estimate future projections of HIV prevalence. We prove global stability of the disease-free equilibrium. We also prove global stability of the endemic equilibrium for the most general case of the model, i.e., which allows for PrEP individuals to default. We include insightful simulations based on recently published South-African data.
\end{abstract}

MSC: $92 \mathrm{D} 30 ; 34 \mathrm{~K} 20$

Keywords: HIV/AIDS model; basic reproduction number; pre-exposure prophylaxis; stability

\section{Introduction}

The HIV/AIDS epidemic continues to be among the most devastating diseases in human history despite the new scientific advances and serious public health interventions. According to UNAIDS data [1], there has been a significant decline and stabilization in the number of new HIV infections since 2012, but Sub-Saharan Africa is still severely affected and more precisely in this region of the world women comprise more than half of all people living with HIV. In particular, in 2016, South Africa had an estimated 7.03 million of people living with HIV [2], and an incidence rate of up to 4 per 100 women-years [3, 4]. The most significant advance in medical management of HIV infection includes two recommendations [5]. First of all, antiretroviral therapy (ART) should be initiated in everyone living with HIV at any CD4 cell count. The HIV treatment reduces viral load to levels below the limits of detection of the most sensitive clinical assays, resulting in a significant reconstitution of the immune system [6]. The Global AIDS Update 2016 of the Joint United Nations Programme on HIV/AIDS, reports that the global coverage of ART therapy reached approximately $46 \%$ at the end of 2015 . The gains in treatment are largely responsible for a $26 \%$ decline in AIDS-related deaths globally since 2010, from an estimated 1.5 million in

(c) The Author(s) 2018. This article is distributed under the terms of the Creative Commons Attribution 4.0 International License (http://creativecommons.org/licenses/by/4.0/), which permits unrestricted use, distribution, and reproduction in any medium, provided you give appropriate credit to the original author(s) and the source, provide a link to the Creative Commons license, and indicate if changes were made. 
2010, to 1.1 million in 2015. Despite this significant achievement, globally there has been 2 million new infections reported in 2015 [7]. Secondly, the use of daily oral pre-exposure prophylaxis (PrEP) is recommended as a prevention choice for people at high risk of HIV infection. Substantial gaps remain in understanding the trade-offs between costs and benefits of choosing alternative HIV prevention strategies, such as the initiation of PrEP by high-risk uninfected individuals [8]. Following WHO, making PrEP drugs available for safe, effective prevention outside the clinical trial setting is the current challenge. However, it is important to recall and highlight that PrEP is not for everyone: only people who are HIV-negative and at very high risk for HIV infection should take PrEP [9]. In 2015, the Medicines Control Council of South Africa issued a full regulatory approval of PrEP, and the country became the first in Sub-Saharan Africa to include PrEP in its national HIV programme. Globally, female sex workers (FSWs) are 13.5 times more likely to be living with HIV than women in the general population [10]. There are many countries with regulatory approval for PrEP. The European Medicines Agency has also granted market authorization for PrEP to be marketed across the European Union's 28 countries [11].

Mathematical models of the population dynamics of infectious diseases are useful in making forward projections in order to help the public health sector to plan optimally. There is a large literature on mathematical models for communicable diseases. In particular, HIV models that account for the use of PrEP are featured in [12-14]. In [12] for instance, a mathematical model for HIV/AIDS transmission has been proposed, along with a control problem in which the objective was to determine the PrEP strategy that minimizes the number of individuals with pre-AIDS HIV infection, balanced against the costs associated with PrEP. The paper by Mukandavire et al. [13] compares the impact of increasing condom use or HIV PrEP use among sex workers. The authors found that condom promotion interventions should remain the mainstay HIV prevention strategy for FSWs, with PrEP only being implemented once condom interventions have been maximized or to fill prevention gaps where condoms cannot be used. In [14], the authors develop a model of HIV risk and compare HIV-risk estimates before and after the introduction of PrEP to determine the maximum tolerated reductions in condom use with regular partners and clients for HIV risk not to change. With a case study of FSWs in South Africa, in [14] it is found that PrEP is likely to be of benefit in reducing HIV risk, even if reductions in condom use do occur. The current paper presents also a significant contribution in this regard. Our aim in this paper is to demonstrate the extent to which PrEP can possibly reduce the prevalence of the HIV in a large population such as South Africa, in the presence of treatment. We introduce a model with two stages of infection and we assume that susceptible individuals have access to PrEP to prevent themselves from HIV. Such individuals become exposed to HIV once they stop taking oral PrEP. The model allows for individuals in the asymptomatic phase to move back to the asymptomatic phase after successful treatment.

The remainder of this paper is set up as follows. In Section 2 we present the model. We calculate the basic reproduction number and prove existence of positive solutions. Section 3 covers both global stability of the disease-free and endemic equilibrium. In Section 4 we provide numerical simulations to illustrate our theoretical results and the utility of the model. In Section 5 we offer some concluding remarks. 


\section{The model}

\subsection{Model description}

We consider a population with homogeneous mixing of individuals, of size $N(t)$ at time $t$. The total size $N(t)$ is assumed to be sufficiently large in order to approximate the population as a continuum of points. These are general assumption for modeling with ordinary differential equations; see for instance [15] of Anderson and May. For this model, the population is subdivided into the classes of susceptibles $S(t)$, the asymptomatic phase $I_{1}(t)$ of HIV, the symptomatic phase $I_{2}(t)$, the AIDS patients $A(t)$ and the individuals under PrEP $E(t)$, so that

$$
N(t)=S(t)+I_{1}(t)+I_{2}(t)+A(t)+E(t) .
$$

The functions $S(t), I_{1}(t), I_{2}(t), A(t)$ and $E(t)$ are assumed to be continuous.

We introduce the following parameters that appear in the model equations:

$\mu \quad$ birth and mortality rates by natural causes,

$K$ the size of the total population when disease-free,

$c$ the average number of sexual contacts of one individual with others, per unit time,

$\beta_{1} \quad$ the probability of disease transmission in the asymptomatic phase,

$\beta_{2} \quad$ the probability of disease transmission in the symptomatic phase,

$\phi \quad$ the proportion of susceptible individuals under PrEP,

$\theta$ the proportion of susceptible individuals who default PrEP,

$k_{1} \quad$ progression rate from $I_{1}$ to $I_{2}$,

$k_{2} \quad$ progression rate from the symptomatic phase $I_{2}$ to $A$,

$\alpha \quad$ the rate of transfer from $I_{2}$ to $I_{1}$ due to ARV treatment,

$\delta \quad$ disease induced mortality rate.

Our model is then constructed by considering the appropriate in-flow and out-flow rates of each compartment together with parameters in the list above.

$$
\begin{aligned}
& \frac{d S}{d t}=\mu K-c\left(\beta_{1} I_{1}+\beta_{2} I_{2}\right) S-(\mu+\phi) S+\theta E, \\
& \frac{d I_{1}}{d t}=c\left(\beta_{1} I_{1}+\beta_{2} I_{2}\right) S-\left(\mu+k_{1}\right) I_{1}+\alpha I_{2}, \\
& \frac{d I_{2}}{d t}=k_{1} I_{1}-\left(\mu+k_{2}+\alpha\right) I_{2}, \\
& \frac{d A}{d t}=k_{2} I_{2}-(\mu+\delta) A, \\
& \frac{d E}{d t}=\phi S-(\mu+\theta) E ; \\
& S(0)=S_{0}>0, \quad I_{1}(0)=I_{1,0}>0, \quad I_{2}(0)=I_{2,0}>0, \\
& A(0)=A_{0}>0, \quad E(0)=E_{0}>0 .
\end{aligned}
$$

The model system (2.1) permits a disease-free equilibrium $\Sigma_{0}=\left(\frac{(\mu+\theta) K}{(\mu+\phi+\theta)}, 0,0,0, \frac{\phi K}{(\mu+\phi+\theta)}\right)$ and an endemic equilibrium $\Sigma_{*}=\left(S^{*}, I_{1}^{*}, I_{2}^{*}, A^{*}, E^{*}\right)$ with the coordinates

$$
S^{*}=\frac{\mu K(\mu+\theta)}{(\mu+\theta)(\lambda+\mu)+\mu \phi}
$$




$$
\begin{aligned}
& I_{1}^{*}=\frac{\lambda\left(\mu+k_{2}+\alpha\right) \mu K(\mu+\theta)}{\left[\left(\mu+k_{2}\right)\left(\mu+k_{1}\right)+\mu \alpha\right][(\mu+\theta)(\lambda+\mu)+\mu \phi]}, \\
& I_{2}^{*}=\frac{k_{1} \lambda \mu K(\mu+\theta)}{\left[\left(\mu+k_{2}\right)\left(\mu+k_{1}\right)+\mu \alpha\right][(\mu+\theta)(\lambda+\mu)+\mu \phi]}, \\
& A^{*}=\frac{k_{1} k_{2} \lambda \mu K(\mu+\theta)}{\left[\left(\mu+k_{2}\right)\left(\mu+k_{1}\right)+\mu \alpha\right][(\mu+\theta)(\lambda+\mu)+\mu \phi]}, \\
& E^{*}=\frac{\mu K \phi}{(\mu+\theta)(\lambda+\mu)+\mu \phi},
\end{aligned}
$$

where

$$
\lambda=c\left(\beta_{1} I_{1}^{*}+\beta_{2} I_{2}^{*}\right) .
$$

Following the method expounded in [16] the basic reproduction number of the model is calculated as

$$
\mathcal{R}_{0}=\frac{c(\mu+\theta) K\left(\beta_{1}\left(\mu+k_{2}+\alpha\right)+\beta_{2} k_{1}\right)}{(\mu+\phi+\theta)\left(\left(\mu+k_{1}\right)\left(\mu+k_{2}\right)+\alpha \mu\right)} .
$$

\subsection{Feasible solutions}

Let us introduce the set $\Omega$,

$$
\Omega=\left\{x \in \mathbb{R}^{5} \mid x_{i}>0, i=1,2,3,4,5 \text { and } x_{1}+x_{2}+x_{3}+x_{4}+x_{5}<K\right\} .
$$

Theorem 2.1 Assume that $X(t)$ is a solution of the system (2.1) with $X(0) \in \Omega$. Then $X(t) \in$ $\Omega$ for all $t>0$.

Proof The proof is by contradiction. Let $X(t)$ be a solution of the system (2.1) where $X(0) \in \Omega$. Suppose to the contrary that there exists a $t_{0}>0$ such that $X\left(t_{0}\right) \notin \Omega$. Let $T=$ $\inf \{t>0: X(t) \notin \Omega\}$. Since $\Omega$ is an open set due to continuity of $X(t), T$ is strictly positive.

Choose $a_{0}>0$ sufficiently small in order to have $a_{0} c \beta_{1}<\mu$ and $a_{0} c \beta_{2}<\mu$. Consider the function $V_{1}$ defined by

$$
\begin{aligned}
V_{1}(t)= & \left(S-a_{0} \ln \frac{S}{a_{0}}\right)+\left(I_{1}-\ln I_{1}\right)+\left(I_{2}-\ln I_{2}\right) \\
& +(A-\ln A)+(E-\ln E) .
\end{aligned}
$$

Note that, for every $T<t$, each of the five bracketed terms on the right hand side of equation (2.2) are positive while $\left(S, I_{1}, I_{2}, A, E\right) \in \Omega$.

Now we find an upper bound for the set

$$
G=\left\{V_{1}(t): 0<t<T\right\} .
$$

We note that, for any $0<t<T$,

$$
\begin{aligned}
\dot{V}_{1}(t)= & {\left[\left(1-\frac{a_{0}}{S}\right)\left(\mu K-c\left(\beta_{1} I_{1}+\beta_{2} I_{2}\right) S-(\mu+\phi) S+\theta E\right)\right] } \\
& +\left[\left(1-\frac{1}{I_{1}}\right)\left(c\left(\beta_{1} I_{1}+\beta_{2} I_{2}\right) S-\left(\mu+k_{1}\right) I_{1}+\alpha I_{2}\right)\right]
\end{aligned}
$$




$$
\begin{aligned}
& +\left[\left(1-\frac{1}{I_{2}}\right)\left(k_{1} I_{1}-\left(\mu+k_{2}+\alpha\right) I_{2}\right)\right] \\
& +\left[\left(1-\frac{1}{A}\right)\left(k_{2} I_{2}-(\mu+\delta) A\right)\right]+\left[\left(1-\frac{1}{E}\right)(\phi S-(\mu+\theta) E)\right] \\
= & \mu K-\frac{a_{0}}{S} \mu K-\mu\left(S+I_{1}+I_{2}+A+E\right)-\frac{a_{0}}{S} \theta E+a_{0}(\mu+\phi)+a_{0} c\left(\beta_{1} I_{1}+\beta_{2} I_{2}\right) \\
& -\frac{1}{I_{1}} c\left(\beta_{1} I_{1}+\beta_{2} I_{2}\right) S+\left(\mu+k_{1}\right)-\frac{1}{I_{1}} \alpha I_{2}+\left(\mu+k_{2}+\alpha\right)-\frac{1}{A} k_{2} I_{2}+(\mu+\delta) \\
& -\frac{1}{E} \phi S+(\mu+\theta) \\
\leq & \mu K-\mu\left(I_{1}+I_{2}\right)+a_{0} c\left(\beta_{1} I_{1}+\beta_{2} I_{2}\right)+4 \mu+a_{0}(\mu+\phi)+k_{1}+k_{2}+\alpha+\delta+\theta .
\end{aligned}
$$

Note that by the choice of $a_{0}$ we have

$$
a_{0} c \beta_{1} I_{1}-\mu I_{1}=I_{1}\left(a_{0} c \beta_{1}-\mu\right)<0 \quad \text { and } \quad a_{0} c \beta_{2} I_{2}-\mu I_{2}=I_{2}\left(a_{0} c \beta_{2}-\mu\right)<0 \text {. }
$$

Therefore

$$
\dot{V}_{1}(t) \leq C,
$$

where $C=\mu K+4 \mu+a_{0}(\mu+\phi)+k_{1}+k_{2}+\alpha+\delta+\theta$.

Integrating from 0 to $t$ yields

$$
V_{1}(t)=V_{1}(0)+\int_{0}^{t} \dot{V}_{1}(s) d s \leq V_{1}(0)+C t \leq V_{1}(0)+C T .
$$

However, we note that, for any positive constant $q$,

$$
\lim _{x \rightarrow 0^{+}}\left(x-q \ln \frac{x}{q}\right)=\infty
$$

Now further, due to positivity of the bracketed terms on the right hand side of equation (2.2), it follows that

$$
\lim _{t \rightarrow T} V_{1}(t)=\infty
$$

Equation (2.4) is in conflict with the inequality (2.3). Thus we have arrived at a contradiction.

\section{Stability analysis}

\subsection{Global stability of the disease-free equilibrium}

The following positive numbers are useful in the proof of the global stability of disease-free equilibrium:

$$
\xi_{1}=\mu+k_{2}+\alpha+k_{1} \frac{\beta_{2}}{\beta_{1}}, \quad \xi_{2}=\alpha+\frac{\beta_{2}}{\beta_{1}}\left(\mu+k_{1}\right), \quad \xi_{4}=\left(\mu+k_{1}\right)\left(\mu+k_{2}\right)+\alpha \mu .
$$

Theorem 3.1 If $\mathcal{R}_{0}<1$, then the disease-free equilibrium $\Sigma_{0}$ of system (2.1) is globally asymptotically stable. 
Proof We introduce a number $\Lambda$ by

$$
\Lambda=\frac{(\mu+\theta) K}{\mu+\phi+\theta} .
$$

Assuming that $\mathcal{R}_{0}<1$, it is possible to find positive numbers $\xi_{0}$ and $\xi_{3}$ sufficiently small such as to have the following inequality:

$$
C_{2}=\xi_{0} c \beta_{2} \Lambda+\xi_{3} k_{2}+\xi_{4}\left(\mathcal{R}_{0}-1\right)<0
$$

Using such $\xi_{0}$ and $\xi_{3}$, together with the numbers $\xi_{i}$ introduced already, we define a function $V_{2}$ as follows:

$$
V_{2}(t)=\xi_{0}[K-(S+E)]+\xi_{1} I_{1}+\xi_{2} I_{2}+\xi_{3} A
$$

The time derivative of $V_{2}(t)$ is given by

$$
\begin{aligned}
\dot{V}_{2}(t)= & \xi_{0}\left[-\mu(K-(S+E))+c\left(\beta_{1} I_{1}+\beta_{2} I_{2}\right) S\right]+\xi_{1}\left[c\left(\beta_{1} I_{1}+\beta_{2} I_{2}\right) S-\left(\mu+k_{1}\right) I_{1}+\alpha I_{2}\right] \\
& +\xi_{2}\left[k_{1} I_{1}-\left(\mu+k_{2}+\alpha\right) I_{2}\right]+\xi_{3}\left[k_{2} I_{2}-(\mu+\delta) A\right] .
\end{aligned}
$$

Grouping some terms we have

$$
\dot{V}_{2}(t) \leq C_{0}[K-(S+E)]+C_{1} I_{1}+C_{2} I_{2}+C_{3} A,
$$

where

$$
\begin{aligned}
& C_{0}=-\mu \xi_{0}<0, \\
& C_{1}=\xi_{0} c \beta_{1} \Lambda+\xi_{1} c \beta_{1} \Lambda-\left(\mu+k_{1}\right) \xi_{1}+\xi_{2} k_{1}, \\
& C_{2}=\xi_{0} c \beta_{2} \Lambda+\xi_{1} c \beta_{2} \Lambda-\left(\mu+k_{2}+\alpha\right) \xi_{2}+\xi_{3} k_{2}+\xi_{1} \alpha, \\
& C_{3}=-(\mu+\delta) \xi_{3}<0 .
\end{aligned}
$$

Now we show that the coefficients $C_{1}, C_{2}$ are also negative. Firstly, it is easy to see that

$$
-\left(\mu+k_{1}\right) \xi_{1}+\xi_{2} k_{1}=-\xi_{4}=-\left(\left(\mu+k_{1}\right)\left(\mu+k_{2}\right)+\alpha \mu\right) .
$$

It follows that

$$
C_{1}=\xi_{0} c \beta_{1} \Lambda+\xi_{1} c \beta_{1} \Lambda-\xi_{4}=\xi_{0} c \beta_{1} \Lambda+\xi_{4}\left(\mathcal{R}_{0}-1\right)<0
$$

Further, notice that

$$
-\left(\mu+k_{2}+\alpha\right) \xi_{2}+\xi_{1} \alpha=-\xi_{4} \frac{\beta_{2}}{\beta_{1}} .
$$

Thus, we have

$$
\xi_{1} c \beta_{2} \Lambda-\xi_{4} \frac{\beta_{2}}{\beta_{1}}=\xi_{4}\left(\mathcal{R}_{0}-1\right) .
$$


Therefore,

$$
C_{2}=\xi_{0} c \beta_{2} \Lambda+\xi_{3} k_{2}+\xi_{4}\left(\mathcal{R}_{0}-1\right)<0
$$

confirming that $V_{2}$ is a Lyapunov function. This completes the proof.

\subsection{Global stability of the endemic equilibrium}

We investigate global stability of the endemic equilibrium of model (2.1) in the general case, that is, when $\theta \neq 0$ and in particular case, when $\theta=0$.

Theorem 3.2 Assume that $\mathcal{R}_{0}>1$ and $\theta E^{*}<c \beta_{1} I_{1}^{*} S^{*}$. Then the endemic equilibrium $\Sigma_{*}$ of system (2.1) is globally asymptotically stable.

Proof Consider a function $V_{3}$ of the form

$$
\begin{aligned}
V_{3}(t)= & \left(S-S^{*}-S^{*} \ln \frac{S}{S^{*}}\right)+D_{1}\left(I_{1}-I_{1}^{*}-I_{1}^{*} \ln \frac{I_{1}}{I_{1}^{*}}\right) \\
& +D_{2}\left(I_{2}-I_{2}^{*}-I_{2}^{*} \ln \frac{I_{2}}{I_{2}^{*}}\right)+D_{3}\left(A-A^{*}-A^{*} \ln \frac{A}{A^{*}}\right) \\
& +D_{4}\left(E-E^{*}-E^{*} \ln \frac{E}{E^{*}}\right),
\end{aligned}
$$

where $D_{1}, D_{2}, D_{3}$ and $D_{4}$ are positive constants, to be determined at a later stage.

The (endemic) equilibrium values of the system (2.1) satisfy the following equations:

$$
\begin{aligned}
& \mu K=S^{*}\left(\beta_{1} I_{1}^{*}+\beta_{2} I_{2}^{*}\right) c+(\mu+\phi) S^{*}-\theta E^{*}, \\
& \left(\mu+k_{2}+\alpha\right)=k_{1} \frac{I_{1}^{*}}{I_{2}^{*}}, \\
& \left(\mu+k_{1}\right)=\frac{S^{*}}{I_{1}^{*}}\left(\beta_{1} I_{1}^{*}+\beta_{2} I_{2}^{*}\right) c+\alpha \frac{I_{2}^{*}}{I_{1}^{*}}, \\
& (\mu+\delta)=k_{2} \frac{I_{2}^{*}}{A^{*}} \\
& (\mu+\theta)=\phi \frac{S^{*}}{E^{*}} .
\end{aligned}
$$

The time derivative of $V_{3}(t)$ is given by

$$
\begin{aligned}
\dot{V}_{3}(t)= & c \beta_{1}\left(1-\frac{S^{*}}{S}\right)\left(I_{1}^{*} S^{*}-I_{1} S\right)+c \beta_{2}\left(1-\frac{S^{*}}{S}\right)\left(I_{2}^{*} S^{*}-I_{2} S\right) \\
& +\left(2-\frac{S}{S^{*}}-\frac{S^{*}}{S}\right) S^{*}(\mu+\phi)+\left(1-\frac{I_{1}^{*}}{I_{1}}\right) D_{1} c \beta_{1}\left(I_{1} S-I_{1} S^{*}\right) \\
& +\left(1-\frac{I_{1}^{*}}{I_{1}}\right) D_{1} c \beta_{2}\left(I_{2} S-I_{2}^{*} S^{*} \frac{I_{1}}{I_{1}^{*}}\right)+\left(E-E^{*}\right) \theta\left(1-\frac{S^{*}}{S}\right) \\
& +D_{3}\left(1-\frac{A^{*}}{A}\right) k_{4}\left(I_{2}-I_{2}^{*} \frac{A}{A^{*}}\right)+D_{2}\left(I_{1}-I_{1}^{*} \frac{I_{2}}{I_{2}^{*}}\right)\left(1-\frac{I_{2}^{*}}{I_{2}}\right) k_{3} \\
& +\left(1-\frac{E^{*}}{E}\right) D_{4}\left(S-S^{*} \frac{E}{E^{*}}\right) \phi .
\end{aligned}
$$


Let

$$
x=\frac{S}{S^{*}}, \quad y=\frac{I_{1}}{I_{1}^{*}}, \quad z=\frac{I_{2}}{I_{2}^{*}}, \quad v=\frac{A}{A^{*}}, \quad w=\frac{E}{E^{*}} .
$$

Then (3.2) becomes

$$
\begin{aligned}
\dot{V}_{3}(t)= & S^{*}(\mu+\phi)\left(2-\frac{1}{x}-x\right)+D_{1} c \beta_{1} I_{1}^{*} S^{*}\left(1-\frac{1}{y}\right)(x y-y) \\
& +D_{1} c \beta_{2} I_{2}^{*} S^{*}\left(1-\frac{1}{y}\right)(x z-y) \\
& +D_{2} I_{1}^{*} k_{1}\left(1-\frac{1}{z}\right)(y-z)+D_{3} I_{2}^{*} k_{2}\left(1-\frac{1}{v}\right)(z-v)+\alpha D_{1} I_{2}^{*}\left(1-\frac{1}{y}\right)(z-y) \\
& +D_{4} k_{2}\left(1-\frac{1}{w}\right) \phi S^{*}(x-w)+c \beta_{1} I_{1}^{*} S^{*}\left(1-\frac{1}{x}\right)(1-x y) \\
& +\beta_{2} I_{2}^{*} S^{*}\left(1-\frac{1}{x}\right)(1-x z)+\left(1-\frac{1}{x}\right)(x-1) \theta E^{*} .
\end{aligned}
$$

This equation informs a choice of values for the numbers $D_{i}$, in order to render $V_{3}$ a Lyapunov function. For making our choices, we require the numbers $D_{i}$ to satisfy the following equations.

$$
\begin{aligned}
& \left(D_{1}-1\right)=0, \\
& -D_{2} I_{1}^{*} k_{1}+\alpha D_{1} I_{2}^{*}+D_{3} I_{2}^{*} k_{2}+c \beta_{2} I_{2}^{*} S^{*}=0, \\
& -D_{1} c \beta_{1} I_{1}^{*} S^{*}-D_{1} c \beta_{2} I_{2}^{*} S^{*}+D_{2} I_{1}^{*} k_{1}-\alpha D_{1} I_{2}^{*}+c \beta_{1} I_{1}^{*} S^{*}=0, \\
& -D_{1} c \beta_{1} I_{1}^{*} S^{*}+D_{4} k_{2} \phi S^{*}+\theta E^{*}=0 .
\end{aligned}
$$

This leads us to choose the following $D_{i}$-values:

$$
\begin{aligned}
& D_{1}=1, \quad D_{2}=\frac{c \beta_{2} S^{*} I_{2}^{*}+\alpha I_{2}^{*}}{k_{1} I_{1}^{*}}, \quad D_{3}=\frac{D_{2} k_{1} I_{1}^{*}-\left(c \beta_{2} S^{*} I_{2}^{*}+\alpha I_{2}^{*}\right)}{k_{2} I_{2}^{*}}, \\
& D_{4}=\frac{c \beta_{1} I_{1}^{*} S^{*}-\theta E^{*}}{k_{2} \phi S^{*}} .
\end{aligned}
$$

Substituting back the $D_{i}$ terms in (3.3), we have

$$
\begin{aligned}
\dot{V}_{3}(t)= & S^{*}(\mu+\phi)\left(2-\frac{1}{x}-x\right)+c \beta_{2} S^{*} I_{2}^{*}\left(3-\frac{1}{x}-\frac{x z}{y}-\frac{y}{z}\right) \\
& +\alpha I_{2}^{*}\left(2-\frac{z}{y}-\frac{y}{z}\right)+\left(c \beta_{1} I_{1}^{*} S^{*}-\theta E^{*}\right)\left(3-w-\frac{1}{x}-\frac{x}{w}\right) \leq 0 .
\end{aligned}
$$

This completes the proof.

In particular, we have the following corollary.

Corollary 3.3 If $\theta=0$, then the endemic equilibrium $\Sigma_{*}$ of system (2.1) is globally asymptotically stable for $\mathcal{R}_{0}>1$. 
Table 1 The following parameters values are fixed

\begin{tabular}{lll}
\hline Parameters & Value & Source \\
\hline$\alpha$ & 0.33 & {$[17]$} \\
$k_{1}$ & 0.125 & {$[18]$} \\
$k_{2}$ & 0.1 & {$[17]$} \\
$c$ & 3 & $c f .[19,20]$ \\
$\delta$ & 0.279 & {$[2]$} \\
$\mu$ & $\frac{1}{62.4}$ & {$[2]$} \\
$\phi$ & 0.01 & Nominal \\
$\theta$ & 0.001 & Nominal \\
\hline
\end{tabular}

\section{Numerical simulation}

The model can be used to test the efficiency of a given intervention. In particular, authorities may want to see the effect of, for example, expanding the use of PrEP. Thus, simulations in this context will also be shown.

We illustrate the analytical results by way of numerical simulations with the parameters applicable to South Africa as in Table 1.

\subsection{Details on estimation of parameters}

In $[19,20]$ for instance, the average number of sexual partners per given time denoted by $c$ is determined; values ranging from 1 to 2 for a specific case. In our case we find it convenient to take $c=3$. We expect the inequality $\beta_{1}<\beta_{2}$ to hold since the intensity of disease transmission in the symptomatic phase exceeds that of the asymptomatic phase. In the year 2016, the life expectancy in South Africa was estimated at 62.4 years; see for instance [2]. The mortality rate $\mu$ is simply the inverse of the life expectancy, and thus $\mu=\frac{1}{62.4}$ year $^{-1}$. The parameter $K$ is the size of the population when it is free from HIV. According to [2], in 2016 South Africa had an estimated total population 55.91 million. Thus we consider it reasonable to choose $K=56$ million. We assume that $1 \%$ of the susceptible individuals take PrEP, that is, $\phi=0.01$ and the default rate takes the value $\theta=0.001$.

\subsection{Initial conditions}

For initial conditions, we first refer to the South African statistical release [2] of 2016 in order to do some projections.

Let us denote the time 25 August 2016 by $t_{0}$. We note that

$$
N\left(t_{0}\right)=S\left(t_{0}\right)+I_{1}\left(t_{0}\right)+I_{2}\left(t_{0}\right)+A\left(t_{0}\right)+E\left(t_{0}\right) .
$$

An estimated 7.03 million of the total population were infected with HIV/AIDS in 2016. This number can be split between the classes of $I_{1}\left(t_{0}\right), I_{2}\left(t_{0}\right)$ and $A\left(t_{0}\right)$. We shall then use the parameters listed in Table 1 below to find a suitable equilibrium point to split the numbers between the classes of $I_{1}\left(t_{0}\right), I_{2}\left(t_{0}\right)$ and $A\left(t_{0}\right)$. In this process we keep varying the value of $\beta_{1}$ and $\beta_{2}$ in order to vary the value of the basic reproduction number. This method leads to the following initial values for our simulations:

$$
I_{1,0}=5.11, \quad I_{2,0}=1.43, \quad A_{0}=0.48
$$

We note that in endemic equilibrium,

$$
E^{*}=\frac{\phi}{\mu+\theta} S^{*} .
$$


Therefore, we consider it reasonable to use the initial value

$$
E\left(t_{0}\right)=\frac{1}{50} \frac{\phi}{\mu+\theta} S\left(t_{0}\right)
$$

This consideration leads us to assign initial values to $S_{0}$ and $E_{0}$, and thus our initial state for these two variables are taken as:

$$
S_{0}=46.18, \quad E_{0}=1.12 \text {. }
$$

\subsection{Simulations on the effect of PrEP}

In the following we show the trajectories of $I_{1}(t), I_{2}(t), A(t)$ of the model for $\phi=0.01$ in Figure 1, and in Figure 2 the trajectories of $I_{2}(t)$ for different values of $\phi, \phi=0.01,0.02,0.03$. For the different values of $\phi$, the corresponding value of $\mathcal{R}_{0}$ will be denoted by $\mathcal{R}_{0}(\phi)$.

In both Figure 1 and Figure 2 we have chosen the values: $\beta_{1}=0.000481, \beta_{2}=0.000581$. In Figure 1 we compute the basic reproduction number, $\mathcal{R}_{0}(0.01)=1.401>1$. The trajectories show that the disease is prevailing at the endemic level. We also compute the endemic equilibrium values $I_{1}^{*}=5.28, I_{2}^{*}=1.48$ and $A^{*}=0.50$ (in millions). In Figure 2 , we show the graph of $I_{2}(t)$ with different values of $\phi$. In the case $\phi=0.02$, the basic reproduction number reduces to $\mathcal{R}_{0}(0.02)=1.021$. This is due to increasing uptake of PrEP from 0.01 to 0.02 , and we observe that the increase in the uptake of PrEP has decreased the basic reproduction number and the class of $I_{2}(t)$. The equilibrium value is computed as $I_{2}^{*}=0.12$. The same scenario is also very well observed in the simulation for the case where

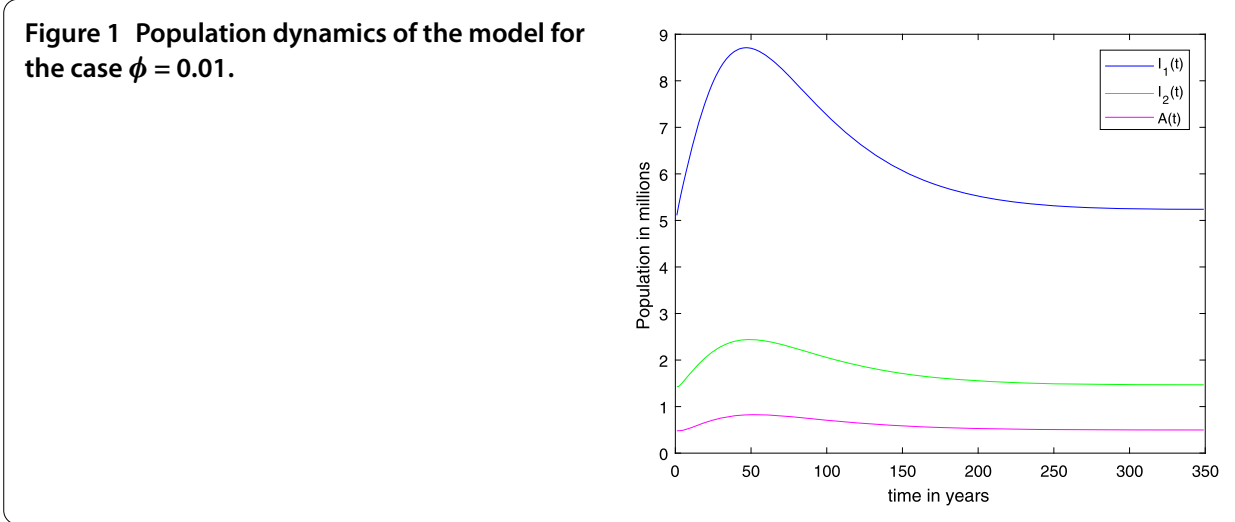

Figure 2 Comparing the class of symptomatic infectives, $l_{2}$, for different values of $\phi$.

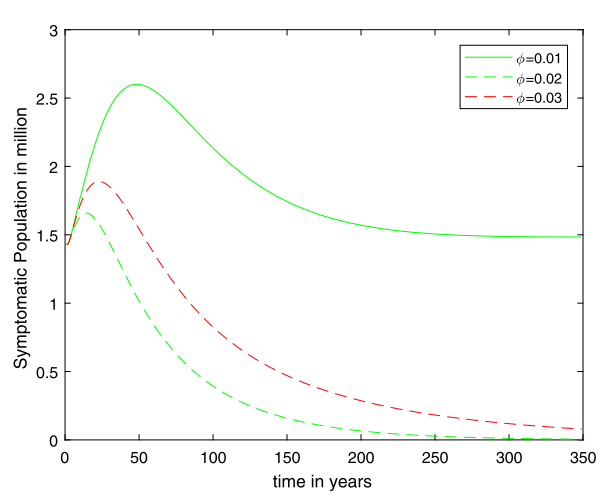


$\phi=0.03$. In this case, the basic reproduction number is found to be below unity, that is, $\mathcal{R}_{0}(0.02)=0.8039$. The class of $I_{2}(t)$ converges to zero. We note $I_{2}^{*}$ is a decreasing function of $\phi$. We note that also the long term (or asymptotic) values of $I_{1}$ and $A$ are decreasing functions of $\phi$.

\section{Concluding remarks}

In this paper, we have investigated a model describing the population dynamics of HIV/AIDS including treatment and pre-exposure prophylaxis (PrEP) in the context of South Africa. We proved global stability of disease-free and endemic equilibria, Theorem 2.1 and Theorem 3.2, respectively. Our analytical results and our sample simulations are quite meaningful as we work with the current HIV trend in South Africa. We showed the substantial impact that treatment has on the incidence, prevalence and mortality due to AIDS. Managing HIV with early treatment can decrease transmission and possibly decrease the number of AIDS-related deaths. Our model quantifies how the use of PrEP potentially reduces the number of new HIV infections, and this has been well observed in the sample simulations. South Africa has a wide range of its population being exposed to HIV. Its high-risk sections of the population include adolescent girls and young women, sex workers, men who have sex with men (MSM), discordant couples and truckers, all of whom face various barriers to access including stigma, criminalization and lack of supportive service delivery infrastructure [21]. If they are to be the focal point for PrEP, it will be imperative to assess how best to introduce PrEP into programmes where these high-risk sections of the population can be supported [22].

Competing interests

The authors declare that there is no conflict of interest regarding the publication of this paper.

\section{Authors' contributions}

The main idea of this paper was proposed by MUN. MUN did most of the proofs of results, in consultation with PJW, and prepared the first draft. The final form of the paper was a joint effort. Both authors read and approved the final manuscript.

\section{Publisher's Note}

Springer Nature remains neutral with regard to jurisdictional claims in published maps and institutional affiliations.

Received: 15 October 2017 Accepted: 27 December 2017 Published online: 11 January 2018

References

1. Council, SANA: National Strategic Plan on HIV, STIs and TB 2012-2016. South African National AIDS Council, Pretoria (2012)

2. Statistics, S.A.: Mid-year population estimates 2016. Statistical Release P0302

3. Hassan, L, El Houssine, L, Mostafa, R, Abdelilah, K: Optimal control of an epidemic model with a saturated incidence rate. Nonlinear Anal., Model. Control 17(4), 448-459 (2012)

4. Baeten, JM, et al.: Use of a vaginal ring containing dapivirine for HIV-1 prevention in women. N. Engl. J. Med. 375(22), 2121-2132 (2016)

5. World Health Organization: WHO Expands Recommendation on Oral Preexposure Prophylaxis of HIV Infection (PrEP): Policy Brief. WHO, Geneva (2015)

6. Arts, EJ, Hazuda, DJ: HIV-1 antiretroviral drug therapy. Cold Spring Harb. Perspect. Med. 2(4), 1-23 (2012)

7. Joint United Nations Programme on HIV/AIDS: Global AIDS Update 2016. UNAIDS, Geneva (2016)

8. Drabo, EF, Hay, JW, Vardavas, R, Wagner, ZR, Sood, N: A cost-effectiveness analysis of pre-exposure prophylaxis for the prevention of HIV among Los Angeles County: men who have sex with men. Clin. Infect. Dis. 63(11), 1495-1504 (2016)

9. www.aids.gov/hiv-aids-basics/prevention/reduce-your-risk/pre-exposure-prophylaxis. Accessed 20 Sep 2017

10. Kerrigan, D, Wirtz, A, et al.: The Global HIV Epidemics Among Sex Workers. The World Bank Publications (2012)

11. European Medicines Agency: First medicine for HIV pre-exposure prophylaxis recommended for approval in the EU. Accessed 22/06/2017

12. Silva, CJ, Torres, DF: Modeling and optimal control of HIV/AIDS prevention through PrEP (2017). arXiv:1703.06446

13. Mukandavire, Z, Mitchell, KM, Vickerman, P: Comparing the impact of increasing condom use or HIV pre-exposure prophylaxis (PrEP) use among female sex workers. Epidemics 14, 62-70 (2016) 
14. Grant, H, Mukandavire, Z, Eakle, R, Prudden, H, Gomez, GB, Rees, H, Watts, C: When are declines in condom use while using PrEP a concern? Modelling insights from a Hillbrow, South Africa case study. J. Int. AIDS Soc. 20(1), 21744 (2017)

15. Anderson, RM, May, RM: Infectious Diseases of Humans: Dynamics and Control. Oxford University, London (1991)

16. van den Driessche, P, Watmough, J: Reproduction numbers and sub-threshold endemic equilibria for compartmental models of disease transmission. Math. Biosci. 180, 29-48 (2002)

17. Bhunu, CP: Assessing the impact of homelessness on HIV/AIDS transmission dynamics. Cogent Math. 2, 1021602 (2015)

18. Hyman, JM, Li, J, Stanley, EA: The differential infectivity and staged progression models for the transmission of HIV. Math. Biosci. 155, 77-109 (1999)

19. Hove-Musekwa, SD, Nyabadza, F: The dynamics of an HIV/AIDS model with screened disease carriers. Comput. Math. Methods Med. 10(4), 287-305 (2009)

20. Nyabadza, F, Mukandavire, Z: Modelling HIV/AIDS in the presence of an HIV testing and screening campaign. J. Theor. Biol. 280(1), 167-179 (2011)

21. Desmond Tutu HIV Foundation: Key populations, key responses: A gap analysis for key populations and HIV in South Africa, and recommendations for the national strategic plan for HIV/AIDS, STIs and TB (2012-2016). http://www.desmondtutuhivfoundation.org.za/documents/Key-Populations-Key-Solutions-report.pdf (2011). Accessed 17 January 2013

22. Eakle, R, Venter, WDF, Rees, H: Pre-exposure prophylaxis for HIV prevention: ready for prime time in South Africa? S. Afr. Med. J. 103(8), 515-516 (2013)

\section{Submit your manuscript to a SpringerOpen ${ }^{\circ}$ journal and benefit from:}

- Convenient online submission

- Rigorous peer review

- Open access: articles freely available online

- High visibility within the field

- Retaining the copyright to your article

Submit your next manuscript at $\boldsymbol{~ s p r i n g e r o p e n . c o m ~}$ 